\section{SP1-21 CORRELATION OF MILD BONE FRAGILITY AND RELATED INDICATORS OF BIOLOGICAL MARKERS OR ULTRASONIC BONE DENSITOMETRY IN A NORMAL JAPANESE POPULATION}

doi:10.1136/jech.2011.142976m.98

N Kuriyama, ${ }^{*}$ E Ozaki, A Yoshikawa, M Shigeta, D Matsui, I Watanabe, K Inoue, Y Watanabe. Kyoto Prefectural University of Medicine, Kyoto, Japan

Introduction Mild bone fragility has the potential to become a significant national problem given the ageing society as it is a precurser of osteoporosis.

Methods Trabecular bone mineral density (BMD), bone elastic modulus (BEM) were measured. We also measured serum bone related antiresorptive markers (eg, calcium), bone absorption markers (TRACP-5b, pyridinoline) and bone formation markers $(\mathrm{BAP})$. Various indicators were compared using $\chi^{2}$ test or trend analysis in the nominated groups.

Results We recruited 348 participants (mean age 45.3 years). We divided subjects into four subgroups: Group C $(n=258$, 74.1\%), Group HBD (hypo BMD, $\mathrm{n}=22,6.3 \%$ ), Group HEM (hypo BEM, $\mathrm{n}=15,4.3 \%$ ), and Group DH (dual hypo, $\mathrm{n}=53$, $15.2 \%), 22$ (6.3\%). Significant associations were found between $\mathrm{HBD} / \mathrm{HEM}$ and increased HbA1c. The BAP level was significantly higher in Group HBD than in Group C. TRACP-5b was significantly higher in Group HEM than in Group C $(p<0.05)$. Drinking and smoking were identified as significant risk factors in Groups HEM and DH.

Conclusion Among the groups, approximately 26\% showed mild bone fragility. Group HBD had a correlation with bone formation marker, whereas Group HEM showed a correlation with bone absorption markers. This implies that BMD and BEM seem to reflect different underlying bone metabolism processes. The present results indicate that bone check-up investigations for and medical prevention of osteoporosis are strongly recommended.

\section{SP1-22 CHARACTERISTICS OF POLYMYOSITIS AND DERMATOMYOSITIS PATIENTS RECEIVING FINANCIAL AID FOR TREATMENT IN JAPAN}

doi:10.1136/jech.2011.142976m.99

A Ohta, ${ }^{*}$ M Nishina, M Nagai. Saitama Medical University, Faculty of Medicine, Moroyama, Saitama, Japan

Introduction Polymyositis and Dermatomyositis (PM/DM) are both rare rheumatic and autoimmune diseases manifesting muscle inflammation and progressive weakness, DM includes skin inflammation, and are unknown cause and difficult to treat. PM/DM is designated as one of the intractable diseases by Ministry of Health, Labour and Welfare of Japan. And the government provides those patients a financial aid for the treatment as a specific disease treatment research program. Simultaneously most of the patients are registered to a database, which includes basic information of patients' characteristics. Using this database, we can clarify in detail the characteristics of the PM/DM patients.

Methods In Japan, 6327 PM/DM patients were registered in 2007 They are about $43 \%$ of the patients receiving financial aid by the government. We analysed the age, sex, age at onset, activity in daily life and clinical features.

Results In the registered patients, the number of male is 1735 and the female is 4592 , sex ratio is 0.38 . Age distribution by sex shows the mode age is $65-69$ and 55-59 for male and female respectively. The age at onset of the disease distributes to wide range from $0-4$ to $85-$, the frequency goes up from the age 20 s to the peak at age 50 s, followed by descending trend. The onset age of female is a little younger than that of male.

Conclusion The registered large number of rare disease (PM/ DM) patients' information provided fundamental features of the disease.

\section{SP1-23 CHARACTERISTICS OF SYSTEMIC LUPUS ERYTHEMATOSUS PATIENTS RECEIVING FINANCIAL AID FOR TREATMENT IN JAPAN}

doi:10.1136/jech.2011.142976m.100

M Nagai, ${ }^{*}$ A Ohta, M Nishina. Saitama Medical University, Faculty of Medicine, Moroyama, Saitama, Japan

Introduction Systemic lupus erythematosus (SLE) is one of the most frequent autoimmune diseases. As SLE is unknown cause and difficult to treat, and the patients are forced to have difficult life, SLE is designated as one of the intractable diseases by Ministry of Health, Labour and Welfare of Japan, same as polymyositis and dermatomyositis (PM/DM) we report in another paper, the government provides those patients a financial aid for the treatment as a specific disease treatment research program. Simultaneously most of the patients are registered to a database, which includes basic information of patients' characteristics. Using this database, we can clarify in detail the characteristics of the SLE patients.

Methods In Japan, 21405 SLE patients were registered in 2007. They are about $39 \%$ of the patients receiving financial aid by the government. We analysed the age, sex, age at onset, activity in daily life and clinical features.

Results In the registered patients, the number of male is 2336 and the female is 19069 , sex ratio is 0.12 . Though an age distribution of male patients shows almost constant frequency from age 50 s to 60 s, that of female shows two peaks at age 30 s and 50 s. The age at onset of female SLE increases from teenage and shows one large peak at age 25-29. That of male does not show any significant peak

Conclusion The characteristics observed here by the analysis of twenty thousand registered patients provided fundamental features of the disease. 